\title{
Deletions of the derivative chromosome 9 occur at the time of the Philadelphia translocation and provide a powerful and independent prognostic indicator in chronic myeloid leukemia
}

Brian J. P. Huntly, Alistair G. Reid, Anthony J. Bench, Lynda J. Campbell, Nick Telford, Patricia Shepherd, Jeff Szer, H. Miles Prince, Paul Turner, Colin Grace, Elizabeth P. Nacheva and Anthony R. Green

Updated information and services can be found at:

http://bloodjournal.hematologylibrary.org/cgi/content/full/98/6/1732

Articles on similar topics may be found in the following Blood collections:

Clinical Trials and Observations (2421 articles)

Neoplasia (4117 articles)

Information about reproducing this article in parts or in its entirety may be found online at:

http://bloodjournal.hematologylibrary.org/misc/rights.dtl\#repub_requests

Information about ordering reprints may be found online at:

http://bloodjournal.hematologylibrary.org/misc/rights.dt|\#reprints

Information about subscriptions and ASH membership may be found online at:

http://bloodjournal.hematologylibrary.org/subscriptions/index.dtl 


\section{Deletions of the derivative chromosome 9 occur at the time of the Philadelphia translocation and provide a powerful and independent prognostic indicator in chronic myeloid leukemia}

Brian J. P. Huntly, Alistair G. Reid, Anthony J. Bench, Lynda J. Campbell, Nick Telford, Patricia Shepherd, Jeff Szer, H. Miles Prince, Paul Turner, Colin Grace, Elizabeth P. Nacheva, and Anthony R. Green

Chronic myeloid leukemia (CML) is char-
acterized by formation of the $B C R-A B L$
fusion gene, usually as a consequence of
the Philadelphia ( $\mathrm{Ph}$ ) translocation be-
tween chromosomes 9 and 22. Large dele-
tions on the derivative chromosome 9
have recently been reported, but it was
unclear whether deletions arose during
disease progression or at the time of the
Ph translocation. Fluorescence in situ
hybridization (FISH) analysis was used to
assess the deletion status of 253 patients
with CML. The strength of deletion status
as a prognostic indicator was then com-
pared to the Sokal and Hasford scoring systems. The frequency of deletions was similar at diagnosis and after disease progression but was significantly increased in patients with variant $\mathrm{Ph}$ translocations. In patients with a deletion, all $\mathrm{Ph}^{+}$metaphases carried the deletion. The median survival of patients with and without deletions was 38 months and 88 months, respectively $(P=.0001)$. By contrast the survival difference between Sokal or Hasford high-risk and non-high-risk patients was of only borderline significance $(P=.057$ and $P=.034)$. The results indicate that deletions occur at the time of the Ph translocation. An appar- ently simple reciprocal translocation may therefore result in considerable genetic heterogeneity ab initio, a concept that is likely to apply to other malignancies associated with translocations. Deletion status is also a powerful and independent prognostic factor for patients with CML. The prognostic significance of deletion status should now be studied prospectively and, if confirmed, should be incorporated into management decisions and the analysis of clinical trials. (Blood. 2001; 98:1732-1738)

๑) 2001 by The American Society of Hematology

\section{Introduction}

Chronic myeloid leukemia (CML) is a clonal hematologic malignancy that results from transformation of a multipotent hemopoietic stem cell. ${ }^{1-3}$ The molecular hallmark of CML is the formation of a $B C R-A B L$ fusion gene, usually formed as a consequence of the Philadelphia $(\mathrm{Ph})$ translocation involving chromosomes 9 and $22 .{ }^{4-6} B C R-A B L$ plays a pivotal role in the pathogenesis of CML and its formation is likely to represent the initiating event. In support of this concept transgenic and retroviral transduction studies have demonstrated that expression of $B C R-A B L$ in murine bone marrow cells resulted in leukemia, with some cases closely resembling CML. ${ }^{7-13}$ In one recent transgenic model the leukemia could be reversed by down-regulating $B C R-A B L .{ }^{14}$

Chronic myeloid leukemia is a biphasic disease with an initial chronic phase that is readily controlled. However, this is followed by an ill-defined accelerated phase, and then a terminal blastic phase that resembles an acute leukemia, which is usually refractory to therapy. Transformation to blast crisis is accompanied by secondary cytogenetic changes in about $85 \%$ of cases, ${ }^{15}$ but the molecular basis for this transformation is poorly understood. A number of molecular changes have been identified in a minority of

From the Department of Hematology, University of Cambridge, Cambridge, United Kingdom; Victorian Cancer Cytogenetic Service, St Vincent Hospital, Fitzroy, Australia; Oncology Cytogenetics Service, Christie Hospital, Manchester, United Kingdom; Department of Hematology, Western General Hospital, Edinburgh, United Kingdom; Department of Clinical Hematology and Medical Oncology, Royal Melbourne Hospital, Melbourne, Australia;Department of Hematology, Peter Macallum Cancer Institute, Melbourne, Australia;Department of Hematology, Alfred Hospital, Melbourne, Australia; and Digital Scientific, Cambridge, United Kingdom.

Submitted February 28, 2001; accepted May 15, 2001. cases of blast crisis, including mutations or deletions of $p 53$, $p 16^{I N K A}$, and the retinoblastoma protein, and mutation or overexpression of Ras and EVI-1.1,2 However, none provide a method for prospectively distinguishing those patients who will progress rapidly to blast crisis from those patients whose disease pursues an indolent course.

Treatment options for patients with chronic phase CML currently include hydroxyurea, interferon $\alpha$ (IFN- $\alpha$; with or without cytosine arabinoside), and allogeneic or autologous stem cell transplantation. Recently there has been considerable interest in STI 571, a novel agent specifically designed to inhibit the tyrosine kinase activity of BCR-ABL, and which has shown excellent early results in all phases of the disease. ${ }^{16,17}$ Other inhibitors of BCR$\mathrm{ABL}$ signal transduction such as farnesyl transferase inhibitors are also showing promise. ${ }^{3,18,19}$ Allogeneic transplantation is potentially curative but may be associated with considerable morbidity and mortality, whereas other forms of current treatment are less dangerous but merely delay the onset of blast crisis. One important goal therefore remains the identification at diagnosis of those patients who have a worse prognosis and in whom more intensive

B.J.P.H. is a Medical Research Council (United Kingdom) Clinical Training Fellow. Work in the authors' laboratories is supported by the Leukemia Research Fund and the Kay Kendall Leukemia Fund.

Reprints: Anthony R. Green, Department of Hematology, University of Cambridge, Wellcome Trust/MRC Building, Hills Rd, Cambridge, CB2 2XY, United Kingdom; e-mail: arg1000@cam.ac.uk.

The publication costs of this article were defrayed in part by page charge payment. Therefore, and solely to indicate this fact, this article is hereby marked "advertisement" in accordance with 18 U.S.C. section 1734.

(C) 2001 by The American Society of Hematology 
treatment modalities are justified. Patients with CML show considerable clinical heterogeneity in chronic phase and a number of prognostic scoring systems have been reported, each based on multiple clinical and laboratory parameters at diagnosis. ${ }^{20,21}$ However, these are not sufficiently robust to routinely form the basis of individual management decisions.

We and others have recently reported previously unrecognized deletions adjacent to the $t(9 ; 22)$ breakpoint on the derivative chromosome $9.22-25$ The deletions were large, spanning up to several megabases, displayed variable breakpoints, and usually resulted in genomic loss of sequences from both the chromosome 9 and chromosome 22 sides of the translocation breakpoint. ${ }^{25}$ However, it was not clear whether the deletions arose during disease progression, ${ }^{22}$ as a consequence of genomic instability, ${ }^{26,27}$ or alternatively whether they occurred at the time of the original $\mathrm{Ph}$ translocation. ${ }^{25}$ Moreover, although it was reported that the deletions may be associated with a more rapid onset of blast crisis, the number of patients studied was small and the survival difference complicated by the fact that few patients with deletions had received IFN- $\alpha .{ }^{25}$ Here we present several lines of evidence to show that the deletions occur at the time of the $\mathrm{Ph}$ translocation and thus result in previously unsuspected genetic heterogeneity from the onset of the disease. Furthermore, our results suggest that deletion status at diagnosis is a powerful and independent prognostic indicator for patients with CML.

\section{Patients, materials, and methods}

\section{Patient samples and clinical and laboratory data}

Fixed cytogenetic preparations from cultured bone marrow samples were obtained from 253 patients with CML, diagnosed between January 1989 and March 2000, in the Department of Hematology at Addenbrooke's Hospital, Cambridge, United Kingdom; the Oncology Cytogenetics Service, Christie Hospital, Manchester, United Kingdom; from patients entered into the United Kingdom Medical Research Council (MRC) CML III trial; and patients diagnosed in the Victorian Cancer Cytogenetics Service, St Vincent's Hospital, Melbourne, Australia. The patients were unselected for type of therapy and were treated at various centers in the United Kingdom and Victoria, Australia. Patients in the CML III trial were previously untreated and were randomized to receive either hydroxyurea or IFN..$^{28}$ These patients were unselected for stage of disease. A diagnostic sample was available for analysis in 123 patients, a subsequent chronic phase sample was analyzed in 66 patients, and an accelerated phase blast crisis sample was available for analysis in 27 patients. Paired samples, where both a diagnostic sample and an accelerated phase/blast crisis sample were available, were analyzed in a further 37 patients. Risk categories were determined as previously described. ${ }^{20,21}$ Data for 55 of these patients have previously been reported. ${ }^{25}$

\section{Fluorescent in situ probes and detection systems}

Triple-probe/3-color system. This system (Figure 1) was used as described. ${ }^{29}$ Briefly, it uses 3 probes, each labeled with a separately colored fluorochrome. These are: (1) ASS probe, a 350-kb cosmid contig that contains the ASS and 8604 Met genes, both mapping proximal to the first exon of ABL and labeled with Spectrum Aqua; (2) ABL probe (Vysis, Downers Grove, IL), a 300-kb cosmid contig that contains the $3^{\prime}$ region of the $A B L$ gene (exons 3-11), labeled with Spectrum Orange; and (3) BCR probe (Vysis), an approximately $300-\mathrm{kb}$ contig that begins between exons 13 and 14 of BCR and extends well beyond the M-bcr region, labeled with Spectrum Green.

Dual-fluorescent in situ hybridization BCR-ABL detection system. This system (Figure 1) was used according to the manufacturer's instructions (Qbiogene, Middlesex, United Kingdom). It uses 2 probes: (1) ABL probe, a 600-kb contig spanning the breakpoint region on $\mathrm{ABL}$ labeled with fluorescein isothiocyanate (FITC) and (2) BCR, a 500-kb contig containing the major and minor breakpoint regions of BCR labeled with Texas red.

\section{Digital imaging and analysis}

All fluorescent in situ hybridization (FISH) images were captured and analyzed with a Smart Capture 2 imaging station, (Digital Scientific, Cambridge, United Kingdom). For each detection system up to 50 images were captured automatically for each patient. A minimum of $30 \mathrm{Ph}^{+}$ metaphase cells were analyzed in 247 patients in which the normal chromosome 9 and 22 displayed appropriate signal patterns. In 6 patients, fewer than $30 \mathrm{Ph}^{+}$metaphase cells were available and results were confirmed by additional analysis of 200 interphase nuclei. The derivative chromosome 9 was identified in patients with deletions by the presence of a heterochromatin block on an appropriately sized chromosome by reverse DAPI (4,6 diamidino-2-phenylindole) banding.

\section{Statistical analysis}

All calculations were performed with the SPSS statistical package (SPSS, Chicago IL). Medians and interquartile ranges were calculated for age and clinical and laboratory findings at diagnosis for patients with and without
Figure 1. Analysis of $\mathrm{Ph}^{+}$metaphase cells with or without a deletion of the derivative chromosome 9 using the triple-probe or D-FISH systems. Each panel shows a cartoon of the expected hybridization signals together with a partial metaphase. Using the triple-probe system $(A, B)$, a blue signal is absent from the derivative chromosome 9 (der 9 ) in a cell carrying a deletion of that chromosome (B). Using the D-FISH system (C,D), note the absence of the colocalized red/green signal on the derivative chromosome 9 in a cell carrying a deletion of that chromosome (D).

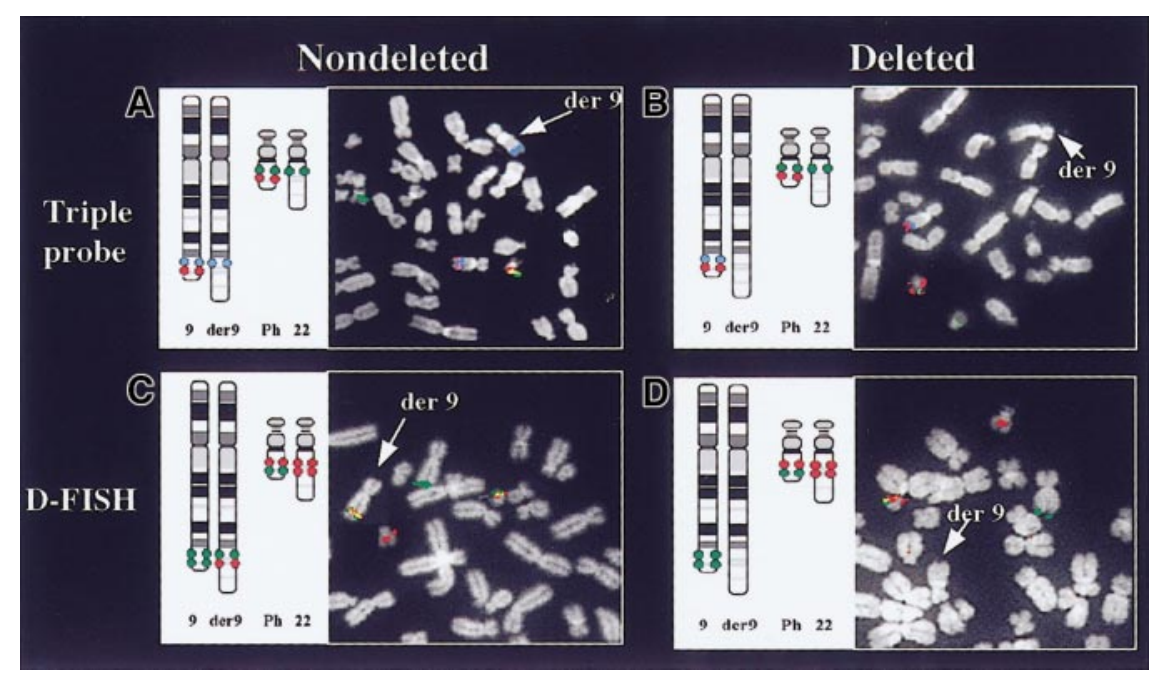


deletions (Table 1) and tested for any significant differences with the Mann-Whitney $U$ test (for continuous variables) or $\chi^{2}$ analysis and Fisher exact tests (for categorical variables). Survival time was calculated from month of presentation to month of death, with a median follow-up of 34 months (range, 1-117 months). Patients who died in chronic phase for reasons unrelated to $\mathrm{CML}$ and bone marrow transplant recipients were censored at the time of death and transplantation, respectively. Survival data were calculated with the Kaplan-Meier estimator and significance was assessed with the log-rank test. The analysis was performed both by time censoring at the time of transplantation or death due to causes unrelated to CML and also by excluding these patients from analysis. Patient age, sex, platelet count, percentage of peripheral blood blasts, classical or variant translocation, and initial Hasford and Sokal scores were considered potentially confounding prognostic factors. The respective $P$ values for the univariate analyses were age $(P=.002)$, sex $(P=.44)$, percentage peripheral blood blasts $(P=.001)$, platelets $(P=.1)$, classical/variant translocation $(P=.14)$, initial Sokal group $(P=.04)$, and initial Hasford group $(P=.08)$. Univariate analysis was performed for these variables and those significant at the $P$ less than .2 level were included in a multivariate analysis

Table 1. Patient characteristics at diagnosis

\begin{tabular}{|c|c|c|}
\hline Patient characteristics $(\mathrm{n}=241)$ & $\begin{array}{c}\text { Patients with } \\
\text { deletions }(n=39)\end{array}$ & $\begin{array}{c}\text { Patients without } \\
\text { deletions }(n=202)\end{array}$ \\
\hline $\operatorname{Sex}(M / F)$ & $23 / 16$ & $110 / 92$ \\
\hline Median age, y (interquartile range) & $50(35-59)$ & $47(37-57)$ \\
\hline No. (\%) with splenomegaly & $24(65 \%)$ & $120(60 \%)$ \\
\hline \multicolumn{3}{|l|}{ Median hemoglobin g/L } \\
\hline (interquartile range) & $100.0(83.5-120.5)$ & $114.5(97.7-129.2)$ \\
\hline \multicolumn{3}{|l|}{ Median WCC $\times 10^{9} / \mathrm{L}$ (interquartile } \\
\hline $\begin{array}{l}\text { Median platelet count } \times 10^{9} / \mathrm{L} \\
\quad(\text { interquartile range) }\end{array}$ & $389(286-655)$ & $405(266-606)$ \\
\hline \multicolumn{3}{|l|}{ Median peripheral blood blasts as $\%$} \\
\hline \multicolumn{3}{|l|}{ Sokal score: number of patients } \\
\hline assessed & 37 & 193 \\
\hline High & $14(38 \%)$ & $67(34 \%)$ \\
\hline Intermediate & $10(27 \%)$ & $63(33 \%)$ \\
\hline Low & $13(35 \%)$ & $63(33 \%)$ \\
\hline \multicolumn{3}{|l|}{ Hasford score: number of patients } \\
\hline assessed & 32 & 178 \\
\hline High & $7(22 \%)$ & $39(21 \%)$ \\
\hline Intermediate & $15(48 \%)$ & $69(39 \%)$ \\
\hline Low & $10(30 \%)$ & $70(40 \%)$ \\
\hline \multicolumn{3}{|l|}{ Treatment type } \\
\hline Interferon- $\alpha+/-$ chemotherapy & $15(39 \%)$ & $116(57 \%)$ \\
\hline Oral chemotherapy & $22(56 \%)$ & $81(40 \%)$ \\
\hline Bone marrow transplantation & $12(31 \%)$ & $63(31 \%)$ \\
\hline \multicolumn{3}{|l|}{ Karyotype } \\
\hline Classical Ph & $23(59 \%)$ & 177 (88\%) \\
\hline Variant $\mathrm{Ph}$ & $16(41 \%)$ & $25(12 \%)$ \\
\hline \multicolumn{3}{|l|}{ Phase of disease at diagnosis (\%) } \\
\hline Chronic phase & 37 (95\%) & $191(94.5 \%)$ \\
\hline Accelerated phase & $1(2.5 \%)$ & $6(2.9 \%)$ \\
\hline Blast crisis & $1(2.5 \%)$ & $5(2.6 \%)$ \\
\hline Patients alive (\%) & $6(16 \%)$ & $84(41 \%)$ \\
\hline Censored $^{*}$ & $13(33 \%)$ & $68(34 \%)$ \\
\hline Dead & $20(51 \%)$ & $50(25 \%)$ \\
\hline
\end{tabular}

Treatment and survival data were available for 241 of 253 patients. Information sufficient to perform Sokal and Hasford scores was available in 230 and 210 patients, respectively. Some patients received oral chemotherapy (hydroxyurea or busulphan) and IFN- $\alpha$ prior to bone marrow transplantation and so the sum of the patients in the treatment section is greater than $100 \%$.

WCC indicates white cell count.

*For patients with deletions, 13 patients were censored, 12 of whom were alive and received a bone marrow transplant; 1 patient died from a cause unrelated to $\mathrm{CML}$. For patients without deletions, 68 patients were censored, of whom 63 were alive and received a bone marrow transplant and 5 died from causes unrelated to $\mathrm{CML}$. from which a forward stepping procedure was used to derive the most significant model. The hazard ratio of deletion status as a univariate analysis was then compared to the hazard ratios following adjustment for initial Hasford and Sokal scores and to the hazard ratio for the most significant model.

\section{Results}

Bone marrow samples from 253 patients with CML were studied using the triple-probe ${ }^{29}$ and dual-FISH (D-FISH) systems. ${ }^{30}$ Figure 1 shows the hybridization patterns expected in "normal" $\mathrm{Ph}^{+}$cells and in $\mathrm{Ph}^{+}$cells carrying a deletion of the derivative chromosome 9. Deletions were detected in 39 of 253 patients (15\%) of whom 30 demonstrated deletion of both chromosome 9 and 22 sequences. Deletions of only chromosome 9 sequences were detected in 7 patients, with deletion of only chromosome 22 sequences detected in 2 patients. There was complete concordance between the triple-probe and D-FISH analyses.

\section{Deletions occur at the time of the $\mathrm{Ph}$ translocation}

Deletions of the derivative chromosome 9 may reflect genomic instability during disease progression or may arise at the time of the initial $\mathrm{Ph}$ translocation. We have investigated this issue in several ways. First, patients analyzed in different phases of the disease were found to exhibit virtually identical frequencies of deletions. In the whole cohort, $15 \%$ (39 of 253) of samples carried a deletion. In samples taken at diagnosis, $14 \%$ (22 of 160) carried a deletion compared to $16 \%$ (10 of 64 ) of samples taken following progression to accelerated phase or blast crisis $\left(\chi^{2}, P=.72\right)$. Sequential paired samples were also analyzed, with the second sample taken following disease progression. In 34 of 37 patients the initial chronic phase sample lacked a deletion. In these 34 patients none of $1306 \mathrm{Ph}^{+}$metaphases obtained after disease progression (30 blast crisis, 4 accelerated phase) had acquired a deletion.

Second, deletions were observed in 16 of $41(39 \%)$ patients with a variant translocation compared to 25 of $212(11 \%)$ patients with a classical $\mathrm{Ph}$ translocation $\left(\chi^{2}, P<.001\right)$. Because variant translocations are thought to result from a complex series of recombination events, ${ }^{31}$ this difference is consistent with a model in which each recombination event has a finite probability of resulting in a deletion adjacent to the breakpoint.

Third, if deletions occurred during disease progression it should be possible to identify cells carrying the $\mathrm{Ph}$ translocation but no deletion. We therefore analyzed a total of 1524 metaphases from the 39 patients shown to carry a deletion. In every metaphase both a $\mathrm{Ph}$ translocation and a deletion were demonstrated.

Taken together these data demonstrate that the recombination event producing an apparently reciprocal translocation also results in large genomic deletions. This process gives rise to previously unsuspected genetic heterogeneity and is likely to be widely applicable to other malignancies associated with translocations.

\section{Deletion status is a powerful prognostic indicator in CML}

Survival data were available from 241 patients (39 with and 202 without a deletion). The clinical and laboratory characteristics of patients with and without deletions are shown in Table 1 and are similar for both. However, Kaplan-Meier analysis revealed a striking difference in survival (Figure 2). The estimated median survival time for patients with deletions was 38 months $(95 \% \mathrm{CI}$ [confidence interval], 36.5-39.4) compared to 88 months (95\% CI, 

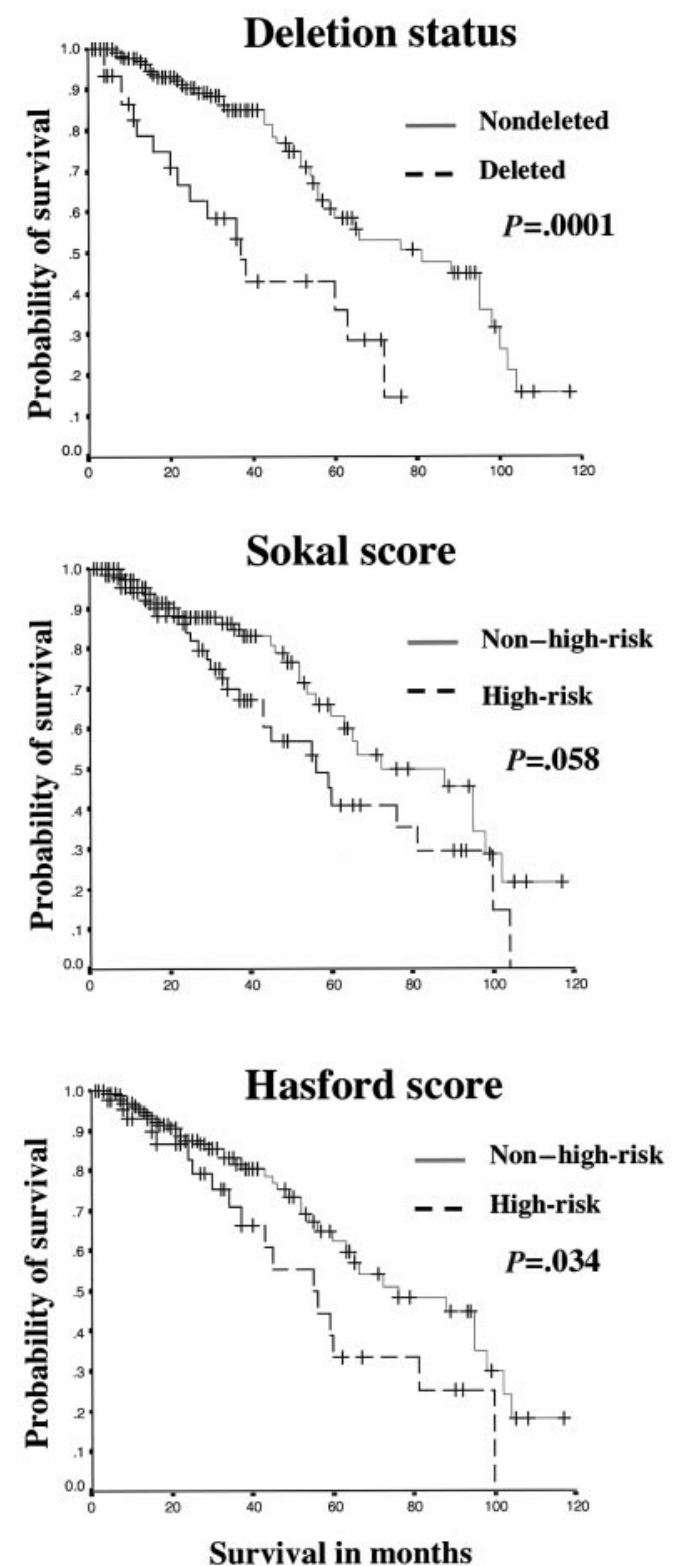

Figure 2. Kaplan-Meier analysis showing prognostic significance of deletion status, Sokal score, and Hasford score. Non-high-risk refers to the combined lowand intermediate-risk groups in the Sokal or Hasford scoring systems. Calculations were performed using data for 210 patients and the significance of survival differences assessed by log-rank analysis. Patients who underwent stem cell transplantation and patients who died of causes unrelated to CML were censored at the time of the procedure.

64-111) for patients without deletions, with a similar median follow-up time for each group (31 versus 34 months, respectively). This difference in survival time was highly significant by log-rank analysis $(P=.0001$; Figure 2$)$. Exclusion of patients who received an allogeneic stem cell transplant $(n=75)$ or were censored due to death unrelated to CML $(\mathrm{n}=6)$ resulted in an increase in the level of significance (log-rank, $P<.0001$ ). This survival difference remained highly significant when the 55 previously reported patients were excluded from the analysis (estimated median survival 81 months for patients without deletions versus 53 months for patients with deletions, $P=.007)$. Hazard ratios for patients with deletions compared to those without are shown in Table 2. These were calculated for deletion status alone, for deletion status after correction for initial Hasford and Sokal scores, and for deletion status following correction for initial blast count and age, the only 2 variables to provide additional prognostic value in the multivariate model constructed. The hazard ratios are all very similar, ranging from 3.0 to 3.2 , indicating the independence of deletion status as a prognostic factor.

Multivariate analysis using a forward stepping model showed the prognostic importance of deletion status remained after adjusting for age, sex, percentage of peripheral blood blasts, platelet count, and initial Sokal and Hasford scores (Table 2). Two potentially confounding variables were further investigated. First, fewer patients with a deletion had received treatment with IFN- $\alpha$ (Table 1). However, this does not account for the observed survival differences because analysis of the subgroup of 131 patients who had received IFN- $\alpha$ also demonstrated markedly worse survival of the patients with a deletion compared to those without a deletion (estimated median survival 38 versus 66 months, log-rank, $P=$.0016). Second, a variant $\mathrm{Ph}$ chromosome was more frequent in patients with a deletion and, although controversial, some previous studies have suggested that such patients have a worse prognosis. ${ }^{32,33}$ However this does not account for the survival difference reported here because there was no significant survival difference between patients with classical or variant $\mathrm{Ph}$ translocations (median survival 76 months versus 60 months, log-rank $P=.15$ ). Moreover, analysis of the subgroup of 200 patients with classical $\mathrm{Ph}$ translocations revealed a worse survival for patients with a deletion compared to those without a deletion (median survival 38 versus 81 months, $\log$-rank $P=.002$ ).

The prognostic strength of deletion status, Sokal score, and Hasford score were then compared in 210 patients for whom all the necessary clinical information was available. As shown in Table 3 and Figure 2, Sokal and Hasford high-risk groups had estimated median survivals of 56 and 55 months, respectively, compared to 37 months for patients with deletions. Moreover, in contrast to the striking prognostic significance of deletion status $(P=.0001)$, the survival difference between high-risk and non-high-risk (low plus intermediate risk) patients using either the Sokal or Hasford scoring systems was only of borderline significance $(P=.058$ and $P=.034$, respectively). Because the Hasford score was developed specifically for patients treated with IFN- $\alpha$, we also compared deletion status and Hasford score in the subgroup of 119 patients who had received IFN- $\alpha$. Again there was a striking difference between the prognostic significance of deletion status and Hasford score $(P=.008$ for deletion status versus $P=.028$ for Hasford score).

Deletion status identified a smaller proportion of patients as being high risk (15\%) compared to the Sokal or Hasford scoring systems (37\% and $22 \%$, respectively; Table 3 ). However, patients with deletions were not merely a subset of the Sokal and Hasford high-risk groups because, within this cohort of 210 patients, similar numbers of patients with deletions were found in Sokal and Hasford low-, intermediate-, and high-risk groups. There were 11

Table 2. Comparison of hazard ratios for deletion status following adjustment for other potential prognostic factors

\begin{tabular}{lc}
\hline \multicolumn{1}{c}{ Adjustment } & Hazard ratio $(95 \% \mathrm{Cl})$ \\
\hline Deletion status alone & $3.2(1.7-5.0)$ \\
All significant variables* & $3.0(1.7-5.5)$ \\
Initial Sokal score & $3.0(1.6-4.9)$ \\
Initial Hasford score & $3.0(1.4-4.8)$ \\
\hline
\end{tabular}

*Hazard ratio was derived from the forward stepping procedure following correction for the initial age and percentage peripheral blood blasts (the only two variables to provide additional prognostic value in the multivariate model constructed). 
From www.bloodjournal.org at UCL Library Services on September 15, 2008. For personal use only.

Table 3. Comparison of prognostic significance of deletion status, Sokal score, and Hasford score

\begin{tabular}{|c|c|c|c|c|c|}
\hline \multirow[b]{2}{*}{ Prognostic factor } & \multicolumn{2}{|c|}{ Non-high-risk } & \multicolumn{2}{|c|}{ High-risk } & \multirow[b]{2}{*}{$\begin{array}{l}\text { Survival } \\
\text { difference }\end{array}$} \\
\hline & $\begin{array}{l}\text { Percent of } \\
\text { patients }\end{array}$ & $\begin{array}{l}\text { Median survival } \\
\quad(95 \% \mathrm{Cl})\end{array}$ & $\begin{array}{l}\text { Percent of } \\
\text { patients }\end{array}$ & $\begin{array}{l}\text { Median survival } \\
\qquad(95 \% \mathrm{Cl})\end{array}$ & \\
\hline Sokal & 63 & $72(51-93)$ & 37 & $56(40-72)$ & $P=.058$ \\
\hline Hasford & 78 & $76(49-103)$ & 22 & $55(34-76)$ & $P=.034$ \\
\hline
\end{tabular}

Analysis was performed using data from 210 patients. Median survivals are shown in months with $95 \% \mathrm{Cl}$. Survival differences were assessed by log-rank analysis. Non-high-risk refers to patients lacking a deletion or, in the Sokal and Hasford systems, the combined low- and intermediate-risk groups. High-risk refers to patients with a deletion or to the Sokal and Hasford high-risk groups.

of $78(14 \%), 9$ of $67(13 \%)$, and 12 of $65(18 \%)$ patients, respectively, in the Sokal low-, intermediate-, and high-risk groups and 10 of $80(13 \%), 15$ of $84(18 \%)$, and 7 of $46(15 \%)$ patients in the 3 respective Hasford risk groups. These results agree well with our findings that the hazard ratios associated with deletion status remain virtually unchanged following adjustment for Sokal or Hasford score, implying independence of deletion status as a prognostic factor (Table 2 and above). Consistent with this concept, the Sokal and Hasford scoring systems retain prognostic significance if analysis is restricted to patients without a deletion (Sokal high-risk versus non-high-risk $P=.03$; Hasford high-risk versus non-high-risk $P=.04$ ). The striking prognostic power of deletion status relative to the Sokal and Hasford systems is likely to reflect the fact that deletion status directly detects a molecular event with a critical role in the progression of CML.

\section{Discussion}

Patients with CML display considerable clinical heterogeneity during the chronic phase of the disease, with some individuals progressing rapidly to blast crisis and death, whereas others remain well controlled for many years. The molecular basis for this variability remains obscure. Here we demonstrate that the $\mathrm{Ph}$ translocation event itself can give rise to considerable genetic heterogeneity in the form of large deletions of sequences on the derivative chromosome 9 . These results show that the pathogenetic consequences of an apparently "simple" translocation may frequently be more complex than previously realized, a concept that is likely to apply to other malignancies with chromosomal translocations.

It has previously been suggested that deletions of the derivative chromosome 9 may be associated with a worse survival. ${ }^{25}$ However, the number of patients studied was small (55 in total and only 16 of whom had deletions). There was also a much higher proportion of variant $\mathrm{Ph}$ translocations (21 of 55, 38\%) within this cohort than occur randomly. Moreover, the significance of the survival difference was complicated by the fact that few patients with deletions had received IFN- $\alpha$ treatment. Here we provide definitive evidence in a large cohort of patients that deletion status is a powerful prognostic indicator for patients with CML. Our results suggest that the prognostic significance of deletion status is much more potent than the widely used Sokal and Hasford scoring systems. This observation has several important clinical consequences. Allogeneic stem cell transplantation is potentially curative, but the procedure is associated with significant morbidity and mortality, especially in older patients or those with unrelated donors. ${ }^{34}$ By contrast IFN- $\alpha$, with or without cytosine arabinoside, is much less dangerous and is effective at controlling chronic phase, but most patients still progress to blast crisis. ${ }^{28,35,36}$ As a result it has been suggested that Sokal or Hasford low-risk patients aged more than 30 years should receive a trial of IFN- $\alpha$ for up to 2 years prior to consideration of allogeneic transplantation. ${ }^{37,38}$ However, our data demonstrate that approximately one third of patients carrying a deletion fall into the Sokal or Hasford low-risk categories. It will therefore be essential to consider deletion status when weighing the risks associated with different treatment modalities. Because the presence of a deletion is likely to influence the outcome of treatment with new agents such as STI 571 or farnesyl transferase inhibitors, it will also be necessary to consider deletion status when analyzing future clinical trials of both conventional and novel therapies.

Our data also provide an explanation for conflicting previous reports of the prognostic significance of variant $\mathrm{Ph}$ translocations. ${ }^{32,33,39,40}$ Patients with a variant $\mathrm{Ph}$ translocation have a more than 3-fold increase in the frequency of deletions, although patients with a deletion still represent a minority $(39 \%)$ of patients with a variant $\mathrm{Ph}$ translocation. Differences in the proportion of patients carrying a deletion are therefore likely to account for previous controversy concerning the prognostic significance of variant compared to classical $\mathrm{Ph}$ translocations.

What might be the molecular mechanism whereby deletions confer such a poor prognosis? Several models can be envisaged. First, deletions will result in loss of the reciprocal fusion gene, $A B L-B C R$. However, current evidence suggests that this is unlikely to be the critical event because no ABL-BCR protein has been detected $^{41}$ and $A B L-B C R$ expression does not correlate with prognosis as assessed by cytogenetic response. ${ }^{42}$ Second, a deletion on the derivative chromosome 9 may act as a surrogate marker for smaller intronic deletions on the $\mathrm{Ph}$ chromosome, which may influence the level of $B C R-A B L$ expression. Blast crisis is sometimes associated with the acquisition of an additional $\mathrm{Ph}$ chromosome suggesting that $B C R-A B L$ dosage may be important.

A third potential mechanism would involve the loss of one or more genes within the deleted region. Such loss may be sufficient to produce a neoplastic effect (haploinsufficiency) or may require subsequent inactivation of the corresponding normal allele(s). ${ }^{43}$ The deletions are large, extending up to $5.5 \mathrm{Mb}$ on the chromosome 9 side of the translocation breakpoint and up to $17 \mathrm{Mb}$ on the chromosome 22 side of the breakpoint (Sinclair et $\mathrm{al}^{25}$ and B.J.P.H., unpublished data, April, 2001). Both of these regions are gene rich. The chromosome 9 region contains 44 known genes and another 40 predicted genes and the chromosome 22 region contains 138 known genes and a further 91 predicted genes based on genome sequence analysis. ${ }^{44}$ The chromosome 22 region contains 3 known tumor suppressor genes, the chromatin remodeling gene hSNF5/ $I N I I,{ }^{45}$ the neurofibromatosis type 2 gene $N F 2,{ }^{46}$ and $M N 1 / M G C R I-$ $P E N$, a putative tumor suppressor gene associated with sporadic meningioma and rearranged in a myeloproliferative disorder with $\mathrm{t}(12 ; 22) .{ }^{47}$ Both the chromosome 9 and chromosome 22 regions contain a number of other genes encoding transcription factors/ cofactors $\left(P B X 3,^{48} L M X 1 B^{49}\right)$, components of signal transduction 
From www.bloodjournal.org at UCL Library Services on September 15, 2008. For personal use only.

pathways (the serine/threonine phosphatase $P P 2 A,{ }^{50}$ Ras inhibitor $I N F,{ }^{51} \mathrm{lim}$ domain protein kinase $L I M-K 2,{ }^{52} \mathrm{GM}-C S F / I L-3 / I L-5$ receptor common chain $\beta^{53}$ ), or cell cycle control proteins $\left(C D K 9^{54}\right)$. Subset analysis of a large series of deletions of varying sizes will be necessary to identify a critical deleted region and thus prioritize candidate genes for further analysis.

Finally, deletions may represent a consequence of genetic instability within the target cell at the time of the $\mathrm{Ph}$ translocation. In this case the poor prognosis would reflect a predisposition to subsequent additional genetic alterations within the malignant clone. Patients with chronic phase CML do not exhibit genomic instability as assessed by microsatellite analy- sis, ${ }^{55,56}$ but these data do not exclude more subtle levels of genetic instability. It is also worth emphasizing that further layers of complexity may exist because the 4 mechanisms discussed above are not mutually exclusive.

\section{Acknowledgments}

We are grateful to Eleanor Pinto, Toby Prevost, and Sue Richards for statistical advice and to the Adult Leukemia Working Party of the Medical Research Council of the United Kingdom for providing 48 patients for the study.

\section{References}

1. Faderl S, Talpaz M, Estrav Z, O’Brien S, Kuzrock $\mathrm{R}$, Kantarjian $\mathrm{H}$. The biology of chronic myeloid leakemia. N Engl J Med. 1999;341:167-172.

2. Deininger M, Goldman J, Melo J. The molecular biology of chronic myeloid leukemia. Blood 2000; 96:3343-3356.

3. Sawyers C. Chronic myeloid leukemia. N Engl J Med. 1999;340:1330-1340.

4. Bartram C, de Klein A, Hagemeijer A. Translocation of c-abl oncogene correlates with the presence of the Philadelphia chromosome in chronic myelocytic leukaemia. Nature. 1983;306:277280.

5. Groffen J, Stephenson J, Heisterkamp N, de Klein A, Bartram C, Grosveld G. Philadelphia chromosome breakpoints are clustered within a limited region, bcr, on chromosome 22. Cell 1984; 36:93-99.

6. Rowley JD. A new consistent chromosomal abnormality in chronic myelogenous leukaemia identified by quinacrine fluorescence and Giemsa staining. Nature 1973;243:290-293.

7. Daley G, Van Etten R, Baltimore D. Induction of chronic myelogenous leukemia in mice by the p210 bcr/abl gene of the Philadelphia chromosome. Science. 1990;247:824-830.

8. Heisterkamp N, Jenster G, ten Hoeve J, Zovich $D$, Pattengale P, Goffen J. Acute leukemia in bcrab/ transgenic mice. Nature. 1990;344:251-253.

9. Elefanty A, Hariharan I, Cory S. BCR-ABL, the hallmark of chronic myeloid leukaemia in man, induces multiple haemopoietic neoplasms in mice. EMBO J. 1990;9:1069-1078.

10. Voncken J, Kaartinen V, Pattengale P, Germeraad W, Groffen J, Heisterkamp N. BCR/ABL p210 and $p 190$ cause distinct leukemia in transgenic mice. Blood. 1995;86:4603-4611.

11. Zhang X, Ren R. Bcr-abl efficiently induces a myeloproliferative disease and production of excess interleukin-3 and granulocyte-macrophage colony-stimulating factor in mice: a novel model for chronic myelogenous leukaemia. Blood. 1998; 92:3829-3840.

12. Pear $\mathrm{W}$, Miller $\mathrm{J}, \mathrm{Xu} \mathrm{L}$, et al. Efficient and rapid induction of a chronic myelogenous leukemia-like myeloproliferative disease in mice receiving p210 bcr/abl transduced bone marrow. Blood. 1998;92: 3780-3792.

13. Honda H, Oda H, Takahiro S, et al. Development of acute lymphoblastic leukemia and myeloproliferative disorder in transgenic mice expressing p210 bcr/abl: a novel transgenic model for human $\mathrm{Ph}^{1}$-positive leukemias. Blood. 1998;91:20672075.

14. Huettner C, Zhang P, Van Etten R, Tenen D. Reversibility of acute $B$-cell leukemia induced by BCR-ABL1. Nat. Genet. 2000;24:57-60.

15. Bernstein R. Cytogenetics of chronic myelogenous leukemia. Semin Hematol. 1988;25:20-34.

16. Druker B, Sawyers C, Kantarjian H, et al. Activity of a specific inhibitor of the Bcr-Abl tyrosine kinase in the blast crisis of chronic myeloid leukaemia and acute lymphoblastic leukaemia with the Philadelphia chromosome. N Engl J Med. 2001 344:1038-1042.

17. Druker B, Talpaz M, Resta D, et al. Efficacy and safety of a specific inhibitor of the Bcr-Abl tyrosine kinase in chronic myeloid leukaemia. N Engl J Med. 2001;344:1031-1037.

18. Peters D, Hoover R, Gertach M, et al. Activity of the farnesyl protein transferase inhibitor SCH66336 against BCR/ABL-induced murine leukaemia and primary cells from patients with chronic myeloid leukaemia. Blood. 2001;97:14041411.

19. Reichert A, Heisterkamp N, Daley G, Groffen J. Treatment of Bcr/Abl-positive acute lymphoblastic leukaemia in P190 mice with the farnesyl transferase inhibitor SCH66336. Blood. 2001;97:1399 1403.

20. Sokal J, Cox E, Baccarini M. Prognostic discrimination in "good risk" chronic granulocytic leukemia. Blood. 1984;63:1352-1357.

21. Hasford J, Pfirrmann M, Hehlmann R, et al. A new prognostic score for survival of patients with chronic myeloid leukemia treated with interferon alpha. J Natl Cancer Inst. 1998;90:850-858.

22. Grand F, Kulkarni S, Chase A, Goldman JM, Cross NCP. Frequent deletion of hSNF5/INI1, a component of the SWI/SNF complex, in chronic myeloid leukaemia. Cancer Res. 1999;59:38703874.

23. Dewald G, Wyatt W, Silver R. Atypical BCR and ABL D-FISH patterns in chronic myeloid leukemia and their possible role in therapy. Leuk Lymphoma. 1999;34:481-491.

24. Herens C, Tassin F, Lemaire V, et al. Deletion of the $5^{\prime}-A B L$ region: a recurrent anomaly detected by fluorescence in situ hybridisation in about $10 \%$ of Philadelphia-positive chronic myeloid leukaemia patients. $\mathrm{Br}$ J Haematol 2000:110:214-216.

25. Sinclair PB, Nacheva EP, Laversha M, et al. Large deletions at the $t(9 ; 22)$ breakpoint are common and may identify a poor-prognosis subgroup of patients with chronic myeloid leukaemia Blood. 2000;95:738-744.

26. Takeda N, Shibuya M, Maru Y. The BCR-ABL oncoprotein potentially interacts with the xeroderma pigmentosum group B protein. Proc Natl Acad Sc U S A. 1999;96:203-207.

27. Canitrot $\mathrm{Y}$, Lautier D, Laurent $\mathrm{G}$, et al. Mutator phenotype of BCR-ABL transfected $B a / F 3$ cell lines and its association with enhanced expression of DNA polymerase beta. Oncogene. 1999; 18:2676-2680.

28. Allan N, Richards S, Shepherd P, et al. UK Medical Research Council randomised multicentre trial of interferon-alpha for chronic myeloid leukemia: improved survival irrespective of cytogenetic response. Lancet. 1995;345:1392-1397.

29. Sinclair P, Green A, Grace C, Nacheva E. Im- proved sensitivity of $B C R-A B L$ detection: a triple probe three-color fluorescence in situ hybridization system. Blood. 1997;90:1395-1402.

30. Dewald G, Wyatt W, Juneau A, et al. Highly sensitive fluorescence in situ hybridization method to detect double BCR/ABL fusion and monitor response to therapy in chronic myeloid leukemia. Blood. 1998:91:3357-3365.

31. Calabrese G, LS, Franchi P, et al. Complex translocations of the $\mathrm{Ph}$ chromosome and $\mathrm{Ph}$ negative $C M L$ arise from similar mechanisms, as evidenced by FISH analysis. Cancer Genet Cytogenet. 1994;78:153-159.

32. Potter A, Watmore A, Cooke P, Lilleyman J, Sokol R. Significance of non-standard Philadelphia chromosomes in chronic granulocytic leukaemia. Br J Cancer. 1981;44:51-54.

33. Bernstein R, Pinto M, Wallace C, Penfold G, Mendelow $B$. The incidence, type and subsequent evolution of 14 variant $\mathrm{Ph}^{1}$ translocations in 180 South African patients with $\mathrm{Ph}^{1}$ chronic myeloid leukemia. Cancer Genet Cytogenet. 1984;12: 225-228.

34. Gratwohl A, Hermans J, Goldman J, et al. Risk assessment for patients with chronic myeloid leukaemia before allogenic blood or bone marrow transplantation. Lancet. 1998;352:1087-1092.

35. The Italian Cooperative Study Group on Chronic Myeloid Leukemia. Interferon-alpha 2a as compared with conventional chemotherapy for the treatment of chronic myeloid leukemia. N Engl J Med. 1994;330:820-825.

36. Guiholt F, Chastang C, Michalet M, Geurci A, Harouseau J-L, Maloisel F. Interferon alfa $2 b \mathrm{com}$ bined with cytarabine versus interferon alpha alone in chronic myelogenous leukemia. N Engl J Med. 1997;337:223-229.

37. Hehlmann R. Trial of IFN or STI 571 before proceeding to allografting for CML. Leukemia. 2000; 14:1560-1562.

38. Tura S, Kantarjian H, Melo J, Giralt S, Talpaz M Chronic myelogenous leukemia: disease biology and current and future therapeutic strategies. In: Schectner G, Berliner N, Telen M, eds. Hematology 2000. San Francisco: American Society of Hematology, 2000:90-109.

39. Sandberg A. Chromosomes and causation of human cancer and leukemia: the $\mathrm{Ph}^{1}$ and other translocations in CML. Cancer. 1980;46:22212226.

40. De Braekleer M. Variant translocations in chronic myeloid leukemia. Cytogenet Cell Genet. 1987; 44:215-222.

41. Melo J. BCR-ABL gene variants. In: Goldman J, ed. Chronic Myeloid Leukaemia. London, United Kingdom: Baillière Tindall: 1997:203-222.

42. Melo J, Hochhaus A, Yan X-H, Goldman J. Lack of correlation between ABL-BCR expression and response to interferon- $\alpha$ in chronic myeloid leukaemia. Br J Haematol. 1996;92:684-686. 
From www.bloodjournal.org at UCL Library Services on September 15, 2008. For personal use only.

43. Asimakopoulos FA, Green AR. Deletions of chromosome $20 \mathrm{q}$ and the pathogenesis of myeloproliferative disorders. Br J Haematol. 1996;95:219226.

44. Birney E, Bateman A, Clamp M, Hubbard T. Mining the draft human genome. Nature. 2001;409: 827-828.

45. Versteege I, Sevent N, Lange J, et al. Truncating mutations of hSNF5/INI1 in aggressive paediatric cancer. Nature. 1998;394:203-206.

46. Ruttledge M, Sarrazin J, Rangaratnam S, et al. Evidence for the complete inactivation of the NF2 gene in the majority of sporadic meningiomas. Nat Genet. 1994;6:180-184

47. Buijs A, Sherr S, van Baal S, et al. Translocation $(12 ; 22)(p 13 ; q 11)$ in myeloproliferative disorders results in fusion of the ETS-like TEL gene on $12 \mathrm{p} 13$ to the MN1 gene on 22q11. Oncogene. 1995;10:1511-1519.

48. Monica K, Galili N, Nourse J, Saltman D, Cleary
M. PBX2 and $P B X 3$, new homeobox genes with extensive homology to the human proto-oncogene PBX1. Mol Cell Biol 1991;11:6149-6157.

49. Dreyer S, Zhou G, Baldini A, et al. Mutations in $L M X 1 B$ cause abnormal skeletal patterning and renal dysplasia in nail patella syndrome. Nat Genet. 1998;19:47-50.

50. Millward T, Zolnierowicz S, Hemmings B. Regulation of the protein kinase cascades by protein phosphatase 2A.Trends Biochem Sci. 1999;24: 186-191.

51. Colicelli J, Nicolette C, Birchmeier C, Rodgers L, Riggs M, Wigler M. Expression of three mammalian cDNAs that interfere with RAS fuction in Saccharomyces cerevisiae. Proc Natl Acad Sci U S A. 1991;88:2913-2917.

52. Maekawa M, Ishizaki T, Boku S, et al. Signalling from Rho to the actin cytoskeleton through protein kinases ROCK and LIM-kinase. Science. 1999;285:895-898.
53. Dirksen U, Hattenhorst U, Schneider P, et al. Defective expression of granulocyte-macrophage colony-stimulating factor/interleukin-3/interleukin- 5 receptor common $\beta$ chain in children with acute myeloid leukaemia associated with respiratory failure. Blood. 1998;92:1097-1103.

54. Grana X, De Luca A, Sang N, et al. PITALRE, a nuclear CDC2-related protein kinase that phosphorylates the retinoblastoma protein in vitro. Proc Natl Acad Sci U S A. 1994;91:3834-3838.

55. Wada C, Shionoya S, Fujino Y, et al. Genomic instability of microsatellite repeats and its association with the evolution of chronic myelogenous leukemia. Blood. 1994;83:3449-3456.

56. Silly $\mathrm{H}$, Chase A, Mills $\mathrm{K}$, et al. No evidence for microsatellite instability or consistent loss of heterozygosity at selected loci in chronic myeloid leukaemia blast crisis. Leukemia. 1994;8:19231928. 